\title{
Electric field control of spin transport
}

\section{SANGEETA SAHO0*, TAKIS KONTOS*, JÜRG FURER, CHRISTIAN HOFFMANN, MATTHIAS GRÄBER, AUDREY COTTET AND CHRISTIAN SCHÖNENBERGER ${ }^{\dagger}$}

Institut für Physik, Universität Basel, Klingelbergstrasse 82, CH-4056 Basel, Switzerland

*These authors contributed equally to this work

†e-mail: Christian.Schönenberger@unibas.ch

$(0$ pintronics aims to develop electronic devices whose resistance is controlled by the spin of the charge carriers that flow through them ${ }^{1-3}$. This approach is illustrated by the operation of the most basic spintronic device, the spin valve $e^{4-6}$, which can be formed if two ferromagnetic electrodes are separated by a thin tunnelling barrier. In most cases, its resistance is greater when the two electrodes are magnetized in opposite directions than when they are magnetized in the same direction ${ }^{7,8}$. The relative difference in resistance, the so-called magnetoresistance, is then positive. However, if the transport of carriers inside the device is spin- or energy-dependent ${ }^{3}$, the opposite can occur and the magnetoresistance is negative $e^{9}$. The next step is to construct an analogous device to a field-effect transistor by using this effect to control spin transport and magnetoresistance with a voltage applied to a gate $^{10,11}$. In practice though, implementing such a device has proved difficult. Here, we report on a pronounced gate-field-controlled magnetoresistance response in carbon nanotubes connected by ferromagnetic leads. Both the magnitude and the sign of the magnetoresistance in the resulting devices can be tuned in a predictable manner. This opens an important route to the realization of multifunctional spintronic devices.

Early work on spin transport in multiwall carbon nanotubes (MWNTs) with Co contacts showed that spins could propagate coherently over distances as long as $250 \mathrm{~nm}$ (ref. 12). The tunnel magnetoresistance $(\mathrm{TMR})=\left(R_{\mathrm{AP}}-R_{\mathrm{P}}\right) / R_{\mathrm{P}}$, defined as the relative difference between the resistances $R_{\mathrm{AP}}$ and $R_{\mathrm{P}}$ in the antiparallel and parallel magnetization configuration, was found to be positive and amounted to $+4 \%$ in agreement with Jullière's formula for tunnel junctions ${ }^{4,13}$. A negative TMR of about $-30 \%$ was reported later for MWNTs contacted with similar Co contacts ${ }^{14}$. In these experiments, the nanotubes did not show quantum dot behaviour. It has been shown, however, that single-wall carbon nanotubes (SWNTs) and MWNTs contacted with nonferromagnetic metals could behave as quantum dots and FabryPérot resonators ${ }^{15-19}$, in which one can tune the position of discrete energy levels with a gate electrode. From this, one can expect to be able to tune the sign and the amplitude of the TMR in nanotubes, in a similar fashion as predicted originally for semiconductor heterostructures ${ }^{11}$.
In this letter, we report on TMR measurements of MWNTs and SWNTs that are contacted with ferromagnetic electrodes and capacitively coupled to a back-gate ${ }^{20}$. A typical sample geometry is shown in the inset of Fig. 1. As a result of resonant tunnelling, we observe a striking oscillatory amplitude and sign modulation of the TMR as a function of the gate voltage. We have studied and observed the TMR on nine samples (seven MWNTs and two SWNTs) with various tube lengths $L$ between the ferromagnetic electrodes (see the Methods section). We present here results for one MWNT device and one SWNT device.

We first discuss the results of the MWNT device. Figure 1 shows single traces of the linear response resistance $R$ as a function of the magnetic field $H$ at $1.85 \mathrm{~K}$ for two sweep directions and four different gate voltages $V_{\mathrm{g}}$. For all cases, the characteristic hysteretic behaviour of a spin valve appears. On sweeping the magnetic field from -500 to $500 \mathrm{mT}$, the configuration becomes antiparallel between 0 and $100 \mathrm{mT}$, whereas it is always parallel for $|H|>100 \mathrm{mT}$. At $V_{\mathrm{g}}=-3.1 \mathrm{~V}$, for example, $R$ increases from 49.7 to $51.5 \mathrm{k} \Omega$ when the sample switches from the parallel to the antiparallel configuration. This yields a normal positive TMR of $+2.9 \%$. In contrast, at $V_{\mathrm{g}}=-3.3 \mathrm{~V}, R$ switches from $30.5 \mathrm{k} \Omega$ in the parallel configuration to a smaller resistance of $29.5 \mathrm{k} \Omega$ in the antiparallel configuration, yielding an anomalous negative TMR of $-3.5 \%$. Therefore, the sign of the TMR changes with the gate voltage, demonstrating a gate-field-tunable magnetoresistance.

Figure 2 a shows the variation of the TMR in a large $V_{\mathrm{g}}$ window of -5 to $2 \mathrm{~V}$ at temperature $T=1.85 \mathrm{~K}$. The TMR is observed to oscillate relatively regularly between -5 and $+6 \%$ on a gate-voltage scale of $\Delta V_{\mathrm{g}}^{\mathrm{TMR}}=0.4-0.75 \mathrm{~V}$. Two possible mechanisms may account for oscillations in spin transport: quantum interference ${ }^{11}$ and gate-field-induced spin precession by means of the Rashba spin-orbit interaction ${ }^{21}$ proposed in ref. 10. In the latter case, the spin-orbit interaction yields a spin precession that is reflected both in the TMR and the conductance. To lowest order, the spin precession would lead to a TMR $\propto \cos \left(2 m L \beta_{\mathrm{R}} e V_{\mathrm{g}} / \hbar^{2}\right)$, where $L$ is the length of the MWNT, $m$ (e) the electron mass (charge) and $\beta_{\mathrm{R}}$ the Rashba spin-orbit parameter. However, the measured magnitude of $\Delta V_{\mathrm{g}}^{\mathrm{TMR}} \sim 1 \mathrm{~V}$ for one TMR period requires a large $\beta_{\mathrm{R}} \sim 10^{-12} \mathrm{~m}$. Although a $\beta_{\mathrm{R}}$ value of this order of magnitude has been reported in semiconductor heterostructures ${ }^{22}$, this value is unreasonably large for carbon nanotubes, owing to the small 


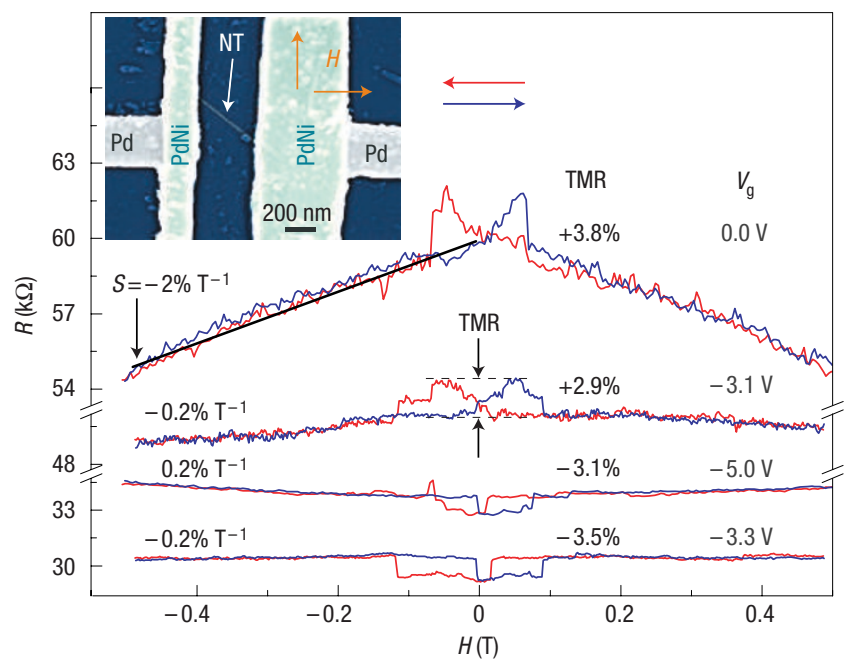

Figure 1 The TMR changes sign with $\boldsymbol{V}_{\mathbf{g}}$. Inset: SEM picture (Philips XL30-FEG) of a carbon nanotube contacted to ferromagnetic PdNi strips. The separation between the contacts along the nanotube amounts to $L=400 \mathrm{~nm}$. The magnetic field $H$ was applied in plane. No qualitative difference has been seen for the field direction parallel and perpendicular to the long axis of the ferromagnetic electrodes. Main panel: Linear response resistance $R$ as a function of $H$ at temperature $T=1.85 \mathrm{~K}$ for different $V_{\mathrm{g}}$. The blue (red) arrow indicates the up (down) magnetic field sweep direction, respectively. The observed amplitude and the sign of the TMR depend on $V_{g}$, but not on the high-field magnetoresistance, which is expressed by $S$ denoting the percentage change of the magnetoresistance with magnetic field (see the Methods section). Note that we extract the maximum possible value for the TMR signal as indicated in the figure.

spin-orbit interaction of carbon leading to an electron $g$-factor close to 2 (refs 19,23,24). We therefore conclude that the mechanism of TMR oscillations is quantum interference instead. To substantiate this, we next compare the TMR gate-voltage scale $\Delta V_{\mathrm{g}}^{\mathrm{TMR}}$ with the corresponding scale $\Delta V_{\mathrm{g}}^{\mathrm{e}}$ for the addition of single electrons.

To resolve single-electron states, the same sample was measured at lower temperatures, that is at $T=300 \mathrm{mK}$ in a different cryostat that is not suitable for TMR studies. A measurement of the differential conductance $\mathrm{d} I / \mathrm{d} V$ as a function of source-drain $V_{\text {sd }}$ and gate voltage $V_{\mathrm{g}}$ at $T=300 \mathrm{mK}$ in a narrow $V_{\mathrm{g}}$ range is shown in Fig. $2 \mathrm{~b}$. It shows the diamond-like pattern characteristic for singleelectron tunnelling in a quantum dot. The visible diamonds vary in size with single-electron addition energies ranging between 0.5 and $0.75 \mathrm{meV}$, in agreement with previous reports on MWNT quantum dots with non-ferromagnetic leads ${ }^{19}$. Comparing with $V_{\mathrm{g}}^{\mathrm{TMR}}$, we see that the average gate-voltage scale $\Delta V_{\mathrm{g}}^{\mathrm{e}}=25 \mathrm{mV}$ associated with one single particle level is much smaller than $\Delta V_{\mathrm{g}}^{\mathrm{TMR}}$. The latter corresponds therefore to the addition of at least 16 electrons rather than one.

A gate-voltage scale that agrees with the TMR signal becomes visible if the linear conductance $G$ at low temperatures is monitored over a wider gate-voltage range. This is shown in Fig. 2c. The singleelectron conductance peaks are strongly modulated in amplitude, leading to a regular beating pattern with the proper gate-voltage scale of $\Delta V_{\mathrm{g}} \approx 0.4 \mathrm{~V}$. Note, the absolute values of $V_{\mathrm{g}}$ cannot be compared with the TMR measurement in Fig. 1a, because the sample was thermally cycled.

Beatings in the amplitude of single-electron resonances are often observed in quantum dot structures and attributed to interference modulation owing to weak disorder. Indeed, as seen in
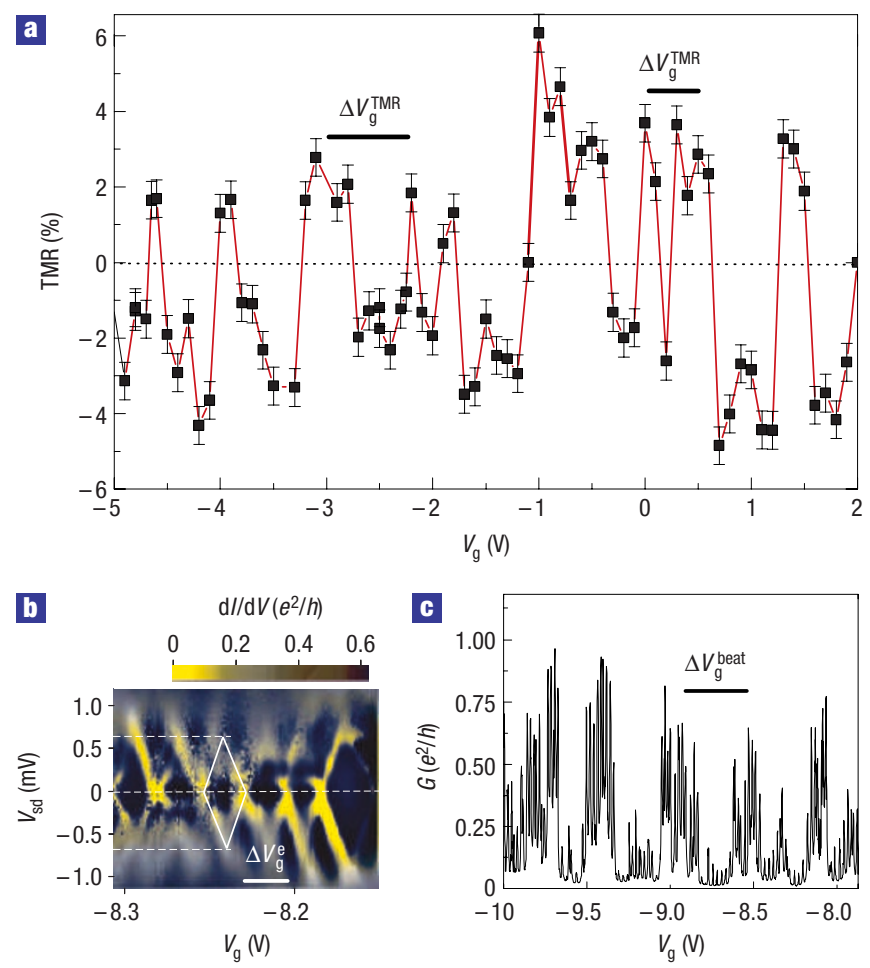

Figure 2 The TMR of the device shown in Fig. 1 oscillates with $\boldsymbol{V}_{\mathbf{g}}$. a, TMR as a function of $V_{\mathrm{g}}$ at $T=1.85 \mathrm{~K}$. The characteristic gate-voltage scale $\Delta V_{\mathrm{g}}^{\text {TMR }}$ of the observed TMR modulation varies between 0.4 and $0.75 \mathrm{~V}$. The bars reflect the error in deducing the TMR signal from $R(B)$ curves, see Fig. 1. b,c, These data were measured in a different cryostat at $300 \mathrm{mK}: \mathbf{b}$, nonlinear differential conductance $\mathrm{d} I / \mathrm{d} V$ as a function of source-drain $V_{\mathrm{sd}}$ and $V_{\mathrm{g}}$ in a narrow $V_{\mathrm{g}}$ interval, corresponding to the addition of six electrons; and $\mathbf{c}$, the linear conductance $G$ over a much wider $V_{g}$ interval.

the $\mathrm{d} I / \mathrm{d} V$ plot of Fig. $2 \mathrm{~b}$, the diamonds do not alternate regularly. In the resonant tunnelling model, one expects each singleparticle peak to contribute negatively to the TMR at sufficiently low temperatures. However, as we have measured the TMR at $T=1.85 \mathrm{~K}$, where the single-particle resonances are already strongly averaged out, the TMR is only sensitive to the average over these peaks, yielding a modulation that follows the envelope function of the single-electron peaks.

The final proof that interference of single-particle levels is that the physical origin for the observed TMR oscillation comes from measurements on SWNTs. Figure 3 shows the conductance $G$ and the TMR of a SWNT device. The quantum dot behaviour is already observed at $1.85 \mathrm{~K}$, whereas this was only evident at $0.3 \mathrm{~K}$ in the MWNT device. This is consistent with the higher energy scales (both single-electron charging energy and level spacing) for SWNTs as compared with MWNTs. As seen in the $\mathrm{d} I / \mathrm{d} V$ plot of Fig. $3 \mathrm{~b}$, the typical single-electron addition energy amounts to $\sim 5 \mathrm{meV}$, whereas it was an order of magnitude smaller in the MWNT device.

In Fig. 3a, the variation of the linear conductance $G$ and the TMR are simultaneously shown for two resonances. First, we observe that the TMR changes sign on each conductance resonance. Furthermore, we see that the line shape of the conductance resonances is symmetric, whereas that of the TMR dips is asymmetric. The jump in the $G\left(V_{\mathrm{g}}\right)$ data at $V_{\mathrm{g}}=4.325 \mathrm{~V}$ is not reproducible, but arises from background charge switching. This jump is absent in a second scan. The amplitude of the TMR 


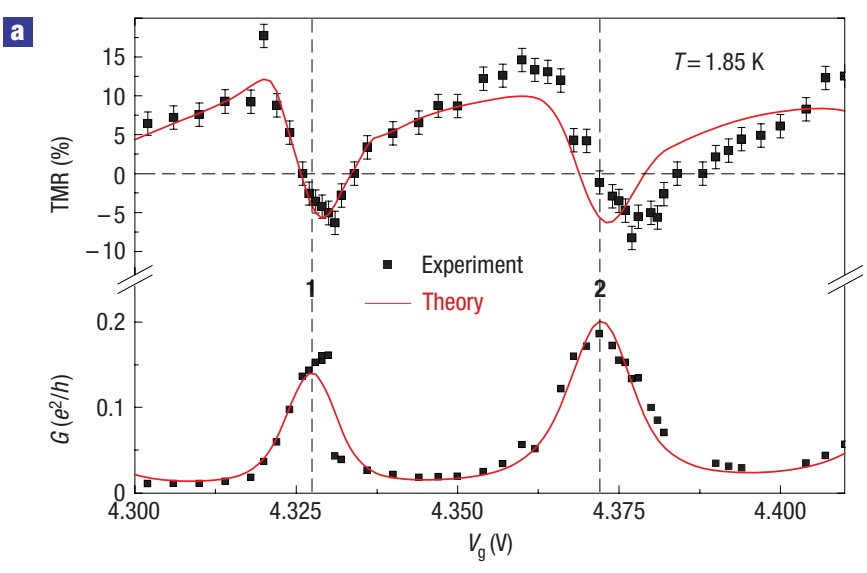

b

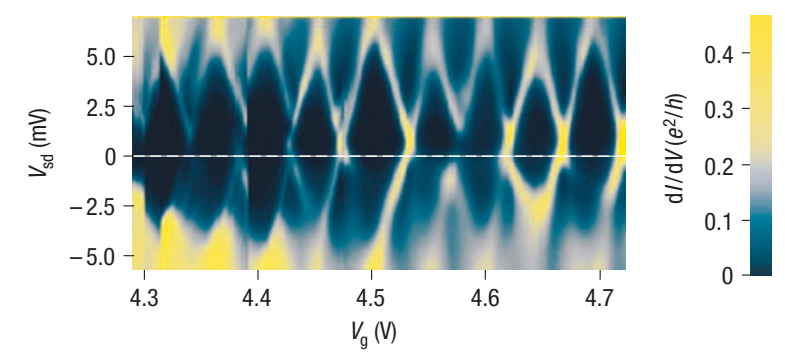

Figure 3 The detailed evolution of the TMR signal for two single-particle resonances. Results for a SWNT device with a contact separation of $L=500 \mathrm{~nm}$ measured at $T=1.85 \mathrm{~K}$. a, Measurement ( $\boldsymbol{a}$ ) of the linear conductance $G$ and the TMR around two resonances and theoretical fit (solid curves) using equation (1) with $P=0.2, \kappa=0.32 \mathrm{meV}$ and $\gamma_{\mathrm{L}}=0.014(0.028) \mathrm{meV}$ and $\gamma_{\mathrm{R}}=0.5(0.85) \mathrm{meV}$ for the left (right) resonance. The bars in a reflect the error in deducing the TMR signal from $R(B)$ curves. $\mathbf{b}$, Plot of the nonlinear differential conductance $\mathrm{d} / / \mathrm{d} V$ as a function of source-drain $V_{\mathrm{sd}}$ and $V_{\mathrm{g}}$. Typical $R(B)$ curves for this F-SWNT-F are shown in Supplementary Information, Fig. S1, as are two representative $R(B)$ curves for N-SWNT-F devices.

ranges from -7 to $+17 \%$, which is a higher amplitude than for the MWNT samples. We think that this might be due to the higher charging energy in SWNTs ${ }^{25}$. Control experiments on two normal-SWNT-ferromagnetic (N-SWNT-F) devices yield an order of magnitude lower signal (see Methods and Supplementary Information), proving that the current in the F-tube-F devices is indeed spin polarized.

The transmission probability through a quantum dot near a resonance can be described by the Breit-Wigner formula. If the quantum dot is coupled to two continua with spin-dependent densities of states, the lifetime of an electron on the quantum dot becomes spin dependent. Therefore, the width of the resonance is different for carriers with up and down spins. In addition, the energy levels $E_{\mathrm{n}}$ of the carbon-nanotube quantum dot acquire a spin-dependent part caused by phases of the reflection amplitudes at the boundaries between the quantum dot and the ferromagnetic electrodes, which are spin dependent ${ }^{26-28}$. The spin-dependent Breit-Wigner transmission probability $T_{\sigma}$ for electrons at energy $E$ with spin orientation $\sigma$ can conveniently be written as

$$
T_{\sigma}=\frac{\Gamma_{\mathrm{L}}^{\sigma} \Gamma_{\mathrm{R}}^{\sigma}}{\left(E-E_{0}^{\sigma}\right)^{2}+\left(\Gamma_{\mathrm{L}}^{\sigma}+\Gamma_{\mathrm{R}}^{\sigma}\right)^{2} / 4},
$$

where $\Gamma_{\mathrm{L}(\mathrm{R})}^{\sigma}=\gamma_{\mathrm{L}(\mathrm{R})}\left(1+\sigma P_{\mathrm{L}(\mathrm{R})}\right)$ denote the spin-dependent $(\sigma= \pm 1)$ coupling to the left (right) ferromagnetic lead, $\gamma_{\mathrm{L}(\mathrm{R})}$ is the bare coupling and $E_{0}^{\sigma}$ is the spin-dependent energy level of the quantum a

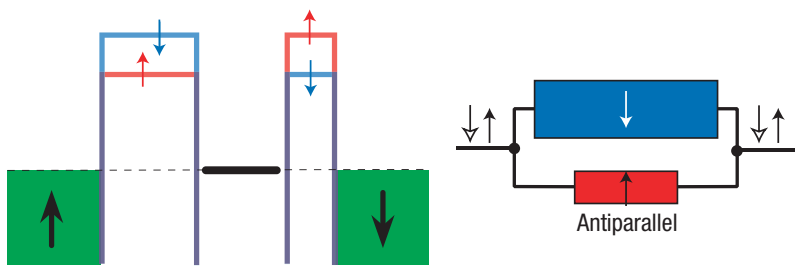

b

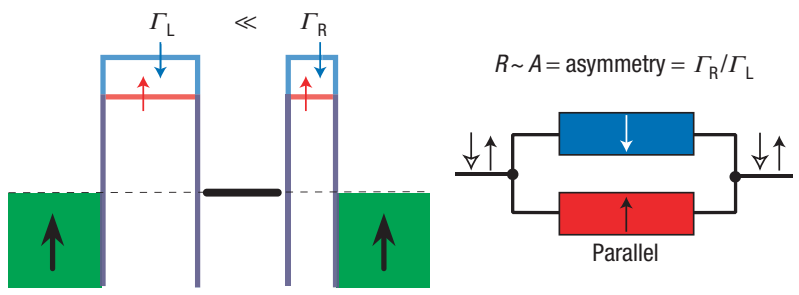

Figure 4 Schematics explaining the observed sign change of the TMR. a, The case for antiparallel magnetization. $\mathbf{b}$, The case for parallel magnetization. The electric resistance of an asymmetric resonant-tunnelling junction (we assume $\Gamma_{\mathrm{R}} \gg \Gamma_{\mathrm{L}}$ ) is proportional to the asymmetry $A=\Gamma_{\mathrm{R}} / \Gamma_{\mathrm{L}}$, where $\Gamma_{\mathrm{R}, \mathrm{L}}$ are the tunnelling rates to the right and left electrode, respectively. If ferromagnetic contacts are used, $\Gamma_{\mathrm{R}, \mathrm{L}}$ become spin-dependent. The rate is increased for the spin direction of the majority carriers, whereas it is decreased for the minority carriers. The two spin directions are colour-coded (red is up spin and blue is down spin). As the electric resistance is spin-dependent, the total resistance $R$ is the parallel circuit of $R^{\uparrow}$ and $R^{\downarrow}$. Whereas $R^{\uparrow}$ and $R^{\downarrow}$ are equal in the parallel configuration, $R^{\uparrow}$ is smaller and $R^{\downarrow}$ is larger in the antiparallel configuration. Owing to the dominance of the smaller resistance in a parallel circuit, $R$ is smaller in the antiparallel compared with the parallel case, corresponding to a negative TMR signal.

dot. Note that the polarization in the leads $P_{\mathrm{L}(\mathrm{R})}$ is measured relative to the spin-quantization axis.

The sign change of the TMR can be predicted with equation (1), provided that the couplings to the leads are asymmetric, see Fig. 4. Off resonance, that is if $\left|E-E_{0}\right| \gg\left(\Gamma_{\mathrm{L}}^{\sigma}+\Gamma_{\mathrm{R}}^{\sigma}\right), T_{\sigma}$ is small and proportional to $\Gamma_{\mathrm{L}}^{\sigma} \Gamma_{\mathrm{R}}^{\sigma}$, yielding the normal positive TMR of $+2 P^{2} /\left(1-P^{2}\right)$ (we assume that $\left.\left|P_{\mathrm{L}}\right|=\left|P_{\mathrm{R}}\right|=P\right)$. On resonance, on the other hand, $T_{\sigma} \propto \Gamma_{\mathrm{L}}^{\sigma} / \Gamma_{\mathrm{R}}^{\sigma}$ (if, for example, $\Gamma_{\mathrm{R}}^{\sigma} \gg \Gamma_{\mathrm{L}}^{\sigma}$ ), yielding an anomalous negative TMR of $-2 P^{2} /\left(1+P^{2}\right)$. This mechanism has already been suggested to explain an observed anomalous TMR in $\mathrm{Ni} / \mathrm{NiO} / \mathrm{Co}$ nanojunctions ${ }^{29}$. Unlike this earlier work, we are able to follow the conductance and the TMR by tuning the energy level $E_{0}$ with the gate voltage $V_{\mathrm{g}}$ and comparing with the model. Whereas the negative TMR can be understood following this line of argument, the explicit shape and, in particular, the asymmetry in the TMR requires a spin-dependent energy level $E_{0}^{\sigma}$ as we will now show. The eigenstate depends on the gate voltage and on the spin direction: $E_{0}^{\sigma}=E_{0}-\epsilon_{\sigma}-\alpha e V_{\mathrm{g}}$, where $\alpha$ is a constant proportional to the gate capacitance, $e$ the unit of charge and $\epsilon_{\sigma}$ the spin-dependent part of the energy level. In the limit of small spin polarization $P_{\mathrm{L}(\mathrm{R})} \ll 1$, one may use the ansatz $\epsilon_{\sigma}=\kappa \sigma\left(P_{\mathrm{L}}+P_{\mathrm{R}}\right)$. We treat $\kappa$ as a fitting parameter, which will be deduced from the experiment. $\kappa$ determines the asymmetry of the TMR signal.

The solid lines in Fig. 3 show fits to the measured conductance $G$ and the TMR using equation (1). As the two resonances are well separated in energy, it is possible to fit them individually. In order to obtain the conductance at finite temperature, we convolved $T_{\sigma}$ with the derivative of the Fermi-Dirac distribution at $1.85 \mathrm{~K}$ and summed over $\sigma$. The following parameters entered the fits: $P=0.2$ and $\kappa=0.32 \mathrm{meV} . \gamma_{\mathrm{L}, \mathrm{R}}$ differ for the two resonances: $\gamma_{\mathrm{L}}=0.014(0.028) \mathrm{meV}$ and $\gamma_{\mathrm{R}}=0.5(0.85) \mathrm{meV}$ for the left (right) resonance, respectively. Using these parameters, a very good 
agreement between theory and experiment is found. Convincing evidence for spin injection in a quantum dot is deduced from the observed asymmetric line shape of the TMR in the SWNT device. The spin imbalance expressed by $\epsilon_{\sigma}$ is substantial, amounting to as much as $\pm 0.13 \mathrm{meV}$, which corresponds to an internal 'exchange field' of $B=2.2 \mathrm{~T}$.

\section{METHODS}

\section{EXPERIMENTAL}

We have developed a reliable scheme to prepare transparent ferromagnetic contacts to MWNTs and SWNTs using the ferromagnetic alloy $\mathrm{Pd}_{1-x} \mathrm{Ni}_{x}$ with $x \sim 0.7$ (ref. 20). Applying standard lithography techniques, a single nanotube is connected to two ferromagnetic PdNi electrodes that are further connected to bonding pads by means of Pd wires. We take advantage of the very good contacting properties of $\mathrm{Pd}$ to nanotubes ${ }^{30}$ and its giant paramagnetism. We have studied and observed the TMR on nine samples (seven MWNTs and two SWNTs) with various tube lengths $L=0.4,0.5,0.8$ and $1 \mu \mathrm{m}$ between the ferromagnetic electrodes. Control samples with $\mathrm{Pd}$ and $\mathrm{PdNi}$ contacts were also fabricated, see below under 'Control experiment'.

\section{STRAY-FIELD EFFECT}

In order to rule out a simple stray-field effect, we compare the high-field magnetoresistance $S$ (defined as a percentage change of the resistance per tesla) with the low-field hysteretic TMR signal. First, we see that the magnitude of the TMR signal of the curves shown in Fig. 1 is to a good approximation constant (to $3.4 \pm 0.4 \%$ ), whereas the background (high-field) magnetoresistance may change by as much as an order of magnitude. Second, the sign change of the TMR from a positive value at $V_{\mathrm{g}}=-3.1 \mathrm{~V}$ to a negative value at $V_{\mathrm{g}}=-3.3 \mathrm{~V}$ is not accompanied by a change in the background $S$. In fact, all possible sign combinations of $S$ and TMR have been observed. As the low-field TMR signal bears no correlation with the background magnetoresistance, we can exclude a stray-field effect from the contacts to the bulk nanotube as the source of the observed hysteretic signal.

\section{CONTROL EXPERIMENT}

In order to ensure that the measured TMR is caused by a coherent spin-polarized current, we have also analysed two devices with asymmetric contacts. One contact (F) is made from the ferromagnetic PdNi alloy of similar composition as was used in the F-SWNT-F devices and the other $(\mathrm{N})$ from the non-ferromagnetic metal Pd. This yields an N-SWNT-F device, which ideally should show no hysteretic signal. On the basis of the noise signal of the resistance measurement, a hysteretic switching signal (if any) must be smaller than $1-1.5 \%$ (see Supplementary Information, Fig. S1). As this is up to ten times smaller than what we have observed in the F-SWNT-F device for similar conductances, any magnetic artefact arising from a single ferromagnetic contact alone must be small, proving that we have observed a spin effect in transport in the F-SWNT-F case.

Received 17 June 2005; accepted 19 September 2005; published 23 October 2005.

References

1. Wolf, S. A. et al. Spintronics: a spin-based electronics vision for the future. Science 294, 1488-1495 (2001).
2. Zorpette, G. The quest for the SPIN transistor. IEEE Spectrum 39, 30-35 (2001).

3. Zutic, I., Fabian, J. \& Das Sarma, S. Spintronics: Fundamentals and applications. Rev. Mod. Phys. 76, 323-410 (2004).

4. Jullière, M. Tunneling between ferromagnetic films. Phys. Lett. A 54, 225-226 (1975).

5. Slonczewski, J. C. Conductance and exchange coupling of two ferromagnets separated by a tunneling barrier. Phys. Rev. B 39, 6995-7002 (1989).

6. Moodera, J. S., Kinder, L. R., Wong, T. M. \& Meservey, R. Magnetoresistance at room temperature in ferromagnetic thin film large tunnel junctions. Phys. Rev. Lett. 74, 3273-3276 (1995).

7. Baibich, M. N., Broto, J. M., Fert, A., Nguyen van Dau, F. \& Petroff, F. Giant magnetoresistance of (001) Fe/(001)Cr magnetic superlattices. Phys. Rev. Lett. 61, 2472-2475 (1988).

8. Binasch, G., Grünberg, P., Saurenbach, F. \& Zinn, W. Enhanced magnetoresistance in layered magnetic structures with antiferromagnetic interlayer exchange. Phys. Rev. B 39, 4828-4830 (1989).

9. George, J. M. et al. Inverse spin-valve-type magnetoresistance in spin engineered multilayered structures. Phys. Rev. Lett. 72, 408-411 (1994).

10. Datta, S. \& Das, B. Electronic analog of the electro-optic modulator. Appl. Phys. Lett. 56, 665-667 (1990).

11. Schäpers, Th., Nitta, J., Heersche, H. B. \& Takayanagi, H. Interference ferromagnet-semiconductorferromagnet spin field-effect transistor. Phys. Rev. B 64, 125314 (2000).

12. Tsukagoshi, K., Alphenaar, B. W. \& Ago, H. Coherent transport of electron spin in a ferromagnetically contacted carbon nanotube. Nature 401, 572-574 (1999).

13. Tedrow, P. M. \& Meservey, R. Spin polarization of electrons tunneling from films of $\mathrm{Fe}, \mathrm{Co}, \mathrm{Ni}$, and Gd. Phys. Rev. B 7, 318-326 (1973).

14. Zhao, B., Mönch, I., Vinzelberg, H., Mühl, T. \& Schneider, C. M. Spin-coherent transport in ferromagnetically contacted carbon nanotubes. Appl. Phys. Lett. 80, 3144-3146 (2002).

15. Tans, S. J. et al. Individual single-wall carbon nanotubes as quantum wires. Nature 386, 474-477 (1997)

16. Bockrath, M. et al. Single-electron transport in ropes of carbon nanotubes. Science 275, 1922-1925 (1997).

17. Liang, W. et al. Fabry-Perot interference in a nanotube electron waveguide. Nature 411, 665-669 (2001).

18. Kong, J., Yenilmez, E., Tombler, T. W., Kim, W. \& Dai, H. Quantum interference and ballistic transmission in nanotube electron waveguides. Phys. Rev. Lett. 87, 106801 (2001).

19. Buitelaar, M. R., Bachtold, A., Nussbaumer, T., Iqbal, M. \& Schönenberger, C. Multiwall carbon nanotubes as quantum dots. Phys. Rev. Lett. 88, 156801 (2002).

20. Sahoo, S., Kontos, T., Schönenberger, C. \& Sürgers, C. Electrical spin injection in multiwall carbon nanotubes with transparent ferromagnetic contacts. Appl. Phys. Lett. 86, 112109 (2005).

21. Bychkov, Yu. A. \& Rashba, E. I. Oscillatory effects and the magnetic susceptibility of carriers in inversion layers. J. Phys. C 17, 6039-6045 (1984).

22. Nitta, J., Akazaki, T., Takayanagi, H. \& Enoki, T. Gate control of spin-orbit interaction in an inverted $\mathrm{In}_{0.53} \mathrm{Ga}_{0.47} \mathrm{As} / \mathrm{In}_{0.52} \mathrm{Al}_{0.48}$ As heterostructure. Phys. Rev. Lett. 78, 1335-1338 (1997).

23. Cobden, D. H., Bockrath, M., McEuen, P. L., Rinzler, A. G. \& Smalley, R. E. Spin splitting and even-odd effects in carbon nanotubes. Phys. Rev. Lett. 81, 681-684 (1998).

24. De Martino, A. \& Egger, R. Rashba spin orbit coupling and spin precession in carbon nanotubes. J. Phys. C 17, 5523-5532 (2005).

25. Barnas, J., Martinek, J., Michalek, G., Bulka, B. R. \& Fert, A. Spin effects in ferromagnetic single-electron transistors. Phys. Rev. B 62, 12363-12373 (2000).

26. Brataas, A., Nazarov, Yu. V. \& Bauer, G. E. W. Finite-element theory of transport in ferromagnet normal metal systems. Phys. Rev. Lett. 84, 2481-2484 (2000).

27. Waintal, X., Myers, E. B., Brouwer, P. W. \& Ralph, D. C. Role of spin-dependent interface scattering in generating current-induced torques in magnetic multilayers. Phys. Rev. B 62, 12317 (2000).

28. Braun, M., König, J. \& Martinek, J. Theory of transport through quantum-dot spin valves in the weak-coupling regime. Phys. Rev. B 70, 195345 (2004).

29. Tsymbal, E. Y., Sokolov, A., Sabirianov, I. F. \& Doudin, B. Resonant inversion of tunneling magnetoresistance. Phys. Rev. Lett. 90, 186602 (2003).

30. Javey, A., Guo, J., Wang, Q., Lundstrom, M. \& Dai, H. Ballistic carbon nanotube field-effect transistors. Nature 424, 654-657 (2003).

\section{Acknowledgements}

We acknowledge fruitful discussions with R. Allenspach, W. Belzig, R. Egger and H. S. J. van der Zant. We thank L. Forró for providing the MWNTs. This work has been supported by the EU RTN network DIENOW, the Swiss National Center (NCCR) on nanoscale science and the Swiss National Science Foundation.

Correspondence and requests for materials should be addressed to C.S.

Supplementary Information accompanies this paper on www.nature.com/naturephysics.

\section{Competing financial interests}

The authors declare that they have no competing financial interests.

Reprints and permission information is available online at http://npg.nature.com/reprintsandpermissions/ 